\title{
WEAK DIMENSION OF GROUP-GRADED RINGS
}

\author{
ANGEL DEL Río
}

\begin{abstract}
We study the weak dimension of a group-graded ring using methods developed in [B1], [Q] and [R]. We prove that if $R$ is a $G$-graded ring with $G$ locally finite and the order of every subgroup of $G$ is invertible in $R$, then the graded weak dimension of $R$ is equal to the ungraded one.
\end{abstract}

One of the main problems in Group-Graded Ring Theory is to see when a group-graded ring has a certain property if it has the similar graded property. Several methods are available in the literature to study these problems. In this paper we use and develop some of these methods to investigate the weak dimension of group-graded rings.

If $R$ is a (graded) ring, then $w \cdot \operatorname{dim}(R)$ (resp, $g r . w . \operatorname{dim}(R)$ ) will denote the (graded) weak dimension of $R$. By [NV, A.I.2.19], $g r . w . \operatorname{dim}(R) \leq w \cdot \operatorname{dim}(R)$. But the equality does not hold in general.

Let $G$ be a group with identity $e$ and $R=\oplus_{g \in G} R_{g}$ a $G$-graded ring.

A separability system $[\mathrm{R}]$ in $R$ is a system $\left\{x^{g}, g \in G\right\}$ of elements in the center of $R_{e}$ satisfying the following axioms:

(SS1) $x^{g}=0$ for almost all $g \in G$ and $\sum_{g \in G} x^{g}=1$.

(SS2) For every $g, h \in G$ and every $r \in R_{h}, r x^{g}=x^{h g} r$.

Note that if $G$ is finite and the order of $G$ is invertible in $R_{e}$, then $R$ has a separability system.

M.D. Rafael has proved in [R] that if $R$ has a separability system, then $g r . w \cdot \operatorname{dim}(R)=w \cdot \operatorname{dim}(R)$.

The main aim of this paper is to give conditions weaker than the existence of a separability system in $R$ which also imply the equality $g r . w . \operatorname{dim}(R)=$ $w \cdot \operatorname{dim}(R)$. Explicity we prove that this holds when for every finitely generated subgroup $H$ of $G$ the $H$-graded ring $R_{(H)}=\oplus_{h \in H} R_{h}$ has a separability system and one of the following conditions hold:

(1) $R$ is gr-regular.

The work of the author has been partially supported by CICYT PB87-0703 and the Institut d'Estudis Catalans 
(2) $R$ is strongly graded.

(3) $G$ is locally finite and the order of each finite subgroup of $G$ is invertible in $R_{\mathrm{e}}$.

When conditions (1) or (2) hold the conclusion follows with an easy argument. The third result needs more complicated methods which involves CohenMontgomery Duality machinery [CM] as well as Rafael's methods [R].

All rings considered in this paper are associative. By left $R$-module we will mean left $R$-module with a spanning set, i.e. for every left $R$-module $M$ we assume $R M=M$.

We will write ${ }_{R} M$ to emphasize that $M$ is a left $R$-module.

For any (graded) $\operatorname{ring} R, R-\bmod (R-g r)$ will denote the category of (graded) left $R$ modules.

The (graded) weak dimension of a (graded) ring $R$ is defined by $R-\bmod$, $(R-g r)$ i.e. given $n \geq 0$, we will say that $w \cdot \operatorname{dim}(R) \leq n(\operatorname{gr} . w . \operatorname{dim}(R) \leq n)$ if for every $M \in R-\bmod ,(R-g r)$ there exists (gr-) flat modules $F_{1}, \ldots, F_{n} \in$ $R-\bmod \left(R-g^{r}\right)$ and an exact sequence

$$
0 \rightarrow F_{n} \rightarrow F_{n-1} \rightarrow \ldots \rightarrow F_{0} \rightarrow M \rightarrow 0 .
$$

Recall that $R$ is (graded) regular if and only if its (graded) weak dimension is 0 .

By [NV, Corollary A.I.2.19], $\operatorname{gr.w.dim}(R) \leq w \cdot \operatorname{dim}(R)$ for every graded ring with unity $R$. But the equality does not hold in general. Indeed, if $R$ is a strongly graded ring with unity, then $g r . w \cdot \operatorname{dim}(R)=w \cdot \operatorname{dim}\left(R_{e}\right)$. In particular, $g r . w \cdot \operatorname{dim}(R[G])=w \cdot \operatorname{dim}(R)$, where $R[G]$ is the group ring over $G$ with base ring $R$ and the natural grading. However, the following well known theorem due to contributions from $M$. Auslander [Au], O.E. Villamayor [V] and I.G. Connell [C] characterizes the regular group rings.

Theorem 1 ([Au], [V], [C]). The group ring $R[G]$ is regular if and only if $R$ is regular, $G$ is locally finite and the order of each finite subgroup of $G$ is invertible in $R$.

The following result due to M.D. Rafael [R] will be used in the sequel.

Theorem $2[\mathrm{R}]$. If the group graded ring $R$ has a separability system, then $g r . w \cdot \operatorname{dim}(R)=w \cdot \operatorname{dim}(R)$.

Proof: See [R; Theorem 3.5].

If $R=\oplus_{g} \in G R_{g}$ is a $G$-graded ring and $H \leq G$ is a subgroup of $G$, then $R_{(H)}$ will denote the $H$-graded ring, $R_{(H)}=\oplus_{h \in H} R_{h}$. 
Proposition 3. Let $R=\oplus_{g \in G} R_{g}$ be a graded ring with unity. Assume that for every finitely generated subgroup $H \leq G, R_{(H)}$ has a separability system. If one of the following conditions holds

(a) $R$ is gr-regular.

(b) $R$ is strongly graded.

Then $g r . w \cdot \operatorname{dim}(R)=w \cdot \operatorname{dim}(R)$.

Proof: (a) Let $x \in R$. Then $x \in R_{(H)}$ for some finitely generated subgroup $H$ of $G$. By Theorem 2, $R_{(H)}$ is regular and hence there exists $y \in R_{(H)}$ such that $x=x y x$.

(b) Since $R$ is strongly graded, then $g r \cdot w \cdot \operatorname{dim}(R)=w \cdot \operatorname{dim}\left(R_{e}\right)=g r . w$. $\operatorname{dim}\left(R_{(H)}\right)=w \cdot \operatorname{dim}\left(R_{(H)}\right)$ for every finitely generated subgroup $H$ of $G$. Finally, note that $R$ is isomorphic to the direct $\operatorname{limit} \lim R_{(H)}$ where $H$ ranges over the finitely generated subgroups of $G$. Then, by $[\overrightarrow{\mathrm{CE}}$, VI Ex. 17], $w \cdot \operatorname{dim}(R) \leq$ $\sup \left\{w . \operatorname{dim}\left(R_{(H)}\right) \mid H \leq G\right.$ f. gen. $\} \leq g r . w \cdot \operatorname{dim}(R)$.

Remarks. (1) Note that if $R$ is strongly graded and for every finitely generated subgroup $H$ of $G, R_{(H)}$ has a separability system, then $G$ is locally finite. Indeed, let $H$ be a finitely generated subgroup of $G . R_{(H)}$ is also strongly graded and, by [R, Lemma 3.2.1], it is also finitely graded. Therefore, $H$ is finite.

(2) The final argument of the proof of Proposition 3.b works whenever gr.w. $\operatorname{dim}\left(R_{(H)}\right) \leq g r \cdot w \cdot \operatorname{dim}(R)$ for every finitely generated subgroup $H$ of $G$. But, unfortunately, this does not hold in general as the following example shows.

Example. Let $k$ be a field and $G=\mathbf{Z}_{2}$ the group with two elements. The polynomial ring $R=k\left[X_{1}, X_{2}, \ldots, X_{n}\right\}$ on $n$ commuting variables $(n \geq 3)$ has a $G$-grading associating to each variable degree 1 . Then $\operatorname{gr.w} \cdot \operatorname{dim}(R)=n$ and $R_{0}=k\left[X_{i} X_{j}, i, j=1,2, \ldots, n\right]$ has infinite weak dimension.

The strong machinery of Cohen-Montgomery Duality Theorem [CM] developed by D. Quinn [Q] and M. Beattie [B1] and [B2] will allow us to obtain a more interesting result.

Let $R=\oplus_{g \in G} R_{g}$ be a $G$-graded ring with unity. Let $M_{G}(R)$ denotes the set of finite row and column matrices over $\mathrm{R}$ with rows and columns indexed by elements of $G$.

If $\alpha \in M_{G}(R)$ and $x, y \in G$, then $\alpha(x, y)$ denotes the entry in the $(x, y)$ position of $\alpha . M_{G}(R)$ is a ring with the matrix ring product: If $\alpha, \beta \in M_{G}(R)$ and $x, y \in G$, then

$$
(\alpha \beta)(x, y)=\sum_{z \in G} \alpha(x, z) \beta(z, y)
$$

$R$ can be embedded in $M_{G}(R)$ by means of a ring homomorphism $R \longrightarrow M_{G}(R)$, that associates $\tilde{r}$ to $r \in R$, where $\tilde{r}(x, y)=r_{x y^{-1}}$ for all $x, y \in G$. 
For every $x \in G, p_{x}$ will denote the $G \times G$-matrix that has 1 in the $(x, x)$ position and zero elsewhere. For each finite subset $X \subseteq G, p_{X}$ will denote the idempotent $p_{X}=\sum_{x \in X} p_{x}$

The smash product $\tilde{R} \# G$ of $R$ was defined by D. Quinn [Q] as the subring of $M_{G}(R)$ generated by $\tilde{R}=\{\tilde{r} \mid r \in R\}$ and $\left\{p_{x} \mid x \in G\right\}$. M. Beattie [B1] defined the generalized smash product as the subring (without unity)

$$
R \# G^{*}=\{\alpha \mid \alpha(x, y)=0 \text { for almost all }(x, y) \in G \times G\}
$$

of $\tilde{R} \# G$. Note that if $G$ is finite, $\tilde{R} \# G=R \# G^{*}$ and if $G$ is infinite, then $R \# G^{*}$ is a left and right essential ideal of $\tilde{R} \# G$.

We list without proof some properties of these rings.

(a) $\left(\tilde{r} p_{x}\right)\left(\tilde{s} p_{y}\right)=\left(r s_{x y^{-1}}\right) \tilde{p} p_{y},(r, s \in R, x, y \in G)$.

(b) $\tilde{r_{g}} p_{x}=p_{g} \tilde{r_{g}},\left(x, g \in G, r_{g} \in R_{g}\right)$.

(c) $\left\{p_{X} \mid X\right.$ finite subset of $\left.G\right\}$ is a set of local units in $R \# G^{*}$ (cf. [Ab]). Thus $R$ is a ring with local units both in the sense of [Ab] and [AM]. Therefore, for every $M \in R \# G^{*}-\bmod$ and each $m \in M$, there exists a finite subset $X \subseteq G$ such that $p_{X} m=m$. Moreover, if $p_{X} m=m \in M$ and $X \subseteq Y \subseteq G$, then $p_{Y} m=m$.

(d) There exists an equivalence of categories between $R-g r$ and $R \# G^{*}-\bmod$ (see [B1, Theorem 2.6]). This equivalence associates the graded left $R$-module $\oplus_{g \in G} R(g)$ to $R \# G^{*}$. Therefore $R \# G^{*}$ is a projective generator in $R \# G^{*}-$ mod.

(e) There is a group homomorphism ${ }^{-}: G \longrightarrow M_{G}(R)$ where $\bar{g}(x, y)=\delta_{g, x^{-1} y}$ for all $g, x, y \in G$. For any $r \in R, g, x \in G, \bar{g}^{-1} \bar{r} \bar{g}=\tilde{r}$ and $\bar{g}^{-1} p_{x} \bar{g}=p_{x_{g}}$. Therefore $\bar{G}=\{\bar{g} \mid g \in G\}$ acts both on $\tilde{R} \# G$ and $R \# G^{*}$ by conjugation.

Let us denote $S=R \# G^{*}$. For every subgroup $H$ of $G, S \bar{H}$ is the subring of $M_{G}(R)$ generated by $S$ and $\bar{H}=\{\bar{h} \mid h \in H\}$. The property (e) implies that $S \vec{H}$ is a skew group ring (without unit if $\mathrm{G}$ is infinite) of $\vec{H}$ over $S$.

Since $S \ddot{H} \in S-\bmod$, then $\left\{p_{X}\{X \subseteq G\right.$ finite $\}$ is also a set of local units of $S \bar{H}$. Therefore, for any $M \in S \bar{H}-\bmod$ and any $m \in M$ there exists $X \subseteq G$ finite such that $p_{X} m=m$.

The following Lemma is a version of Maschke's Theorem.

Lemma 4. Assume that $G$ is locally finite and let $H$ be a finite subgroup of $G$ such that $|H|^{-1} \in R_{e}$. Then every $M \in S \bar{H}-$ mod, is isomorphic to a direct

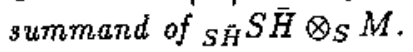

Proof: Let $p: S \bar{H} \otimes_{S} M \longrightarrow M$ be the epimorphism given by $p(\alpha \otimes m)=$ $\alpha m$. We will construct a right inverse of p. Assume $n=|H|$ and define $j: M \longrightarrow S \bar{H} \otimes S M$ as follows: Let $m \in M$ and $X$ a finite subset of $G$ such that $p_{X} m=m$. Then

$$
j(m)=\frac{1}{n} \sum_{h \in H} p_{X} \overline{h^{-1}} \otimes p_{X h^{-}} \bar{h} m
$$


Let us note that $j(m)$ does not depend on the choice of $X$. Indeed, let $X^{t}$ be another finite subset of $G$ such that $p_{X} \cdot m=m$ and consider $Y$ the subgroup of $G$ generated by $H \cup X \cup X^{\prime}$. For every $h \in H$

$$
\begin{aligned}
p_{Y} \overline{h^{-1}} \otimes p_{Y^{-1}} \bar{h} m & =p_{Y} \overline{h^{-1}} \otimes p_{Y} \bar{h} p_{X} m \\
& =p_{Y} \overline{h^{-1}} \otimes p_{Y} p_{X^{-1}} \bar{h} m \\
& =p_{Y} \overline{h^{-1}} \otimes p_{X^{-1}} \bar{h} m \\
& =p_{Y} \overline{h^{-1}} p_{X^{-1}} \otimes p_{X^{-1}} \bar{h} m \\
& =p_{Y} p_{X} \overline{h^{-1}} \otimes p_{X^{-1}} \bar{h} m \\
& =p_{X} \overline{h^{-1}} \otimes p_{X^{-1}} \bar{h} m
\end{aligned}
$$

and similarly $p_{Y} \overline{h^{-1}} \otimes p_{Y^{-1}} \bar{h} m=p_{X^{\prime}} \overline{h^{-1}} \otimes p_{X^{\prime} h-1} \bar{h} m$. It is rather easy to proof that $j$ is an additive homomorphism. Let us prove that $j$ is $S \bar{H}$-linear. Set $g, \tau \in G, \sigma \in H$ and $r \in R_{\tau}$. Assume that $p_{X} m=m \in M$ ( $X$ a finite subset of $G$ ). Consider $Y$ the subgroup of $G$ generated by $X \cup\{g, \tau\}$. We have that $p_{Y} m=m$ and $p_{Y}\left(p_{g} \tilde{r} \bar{\sigma} m\right)=p_{g} \tilde{r} \bar{\sigma} m$. Then

$$
\begin{aligned}
j\left(p_{g} \tilde{r} \tilde{\sigma} m\right) & =\frac{1}{n} \sum_{h \in H} p_{Y} \overline{h^{-1}} \otimes p_{Y h-1} \bar{h} p_{g} \tilde{r} \bar{\sigma} m \\
& =\frac{1}{n} \sum_{h \in H} p_{Y} \overline{h^{-1}} \otimes p_{Y^{-1}} p_{g h^{-1}} \tilde{r} \overline{h \sigma} m \\
& =\frac{1}{n} \sum_{h \in H} p_{Y} \overline{h^{-1}} p_{g k^{-1}} \tilde{r} \otimes p_{Y k^{-1}} \overline{h \sigma} m \\
& =\frac{1}{n} \sum_{h \in H} p_{Y} p_{g} \tilde{r} \overline{h^{-1}} \otimes p_{Y k^{-1}} \overline{h \sigma} m \\
& =p_{g} \tilde{r} \bar{\sigma} \frac{1}{n} \sum_{h \in H} p_{Y} \overline{(h \sigma)^{-1}} \otimes p_{Y^{-1}} \overline{h \sigma} m \\
& =p_{g} \tilde{r} \bar{\sigma} j(m)
\end{aligned}
$$

For $m \in M$ and $p_{X} m=m$ we have

$$
\begin{aligned}
(p \circ j)(m) & =p\left(\frac{1}{n} \sum_{h \in H} p_{X} \overline{h^{-1}} \otimes p_{X h^{-1}} \bar{h} m\right) \\
& =\frac{1}{n} \sum_{h \in H} p_{X} \overline{h^{-1}} p_{X h^{-1}} \bar{h} m \\
& =\frac{1}{n} \sum_{h \in H} p_{X}^{2} \overline{h^{-1}} \bar{h} m=m .
\end{aligned}
$$

Thus $p \circ j=1_{M}$. 
Lemma 5. Assume that $G$ is locally finite and for every finite subgroup $H$ of $G,|H|^{-1} \in R_{e}$. Let $M \in S \bar{G}-\bmod$ such that $S M$ is projective (resp, flat). Then, $S \bar{G} M$ is also projective (resp, flat).

Proof: Let $M \in S \bar{G}-\bmod$ such that ${ }_{S} M$ projective (resp, flat). By (e) $s S$ is projective and flat, thus for every subgroup $H$ of $G,{ }_{s} S \bar{H}$ is also projective and hence $s \bar{H} S \bar{H} \otimes_{S} M$ is also projective (resp, flat). Now, by Lemma $4{ }_{S \bar{H}} M$ is projective (resp, flat) for every finite subgroup $H$ of $G$. For every left (resp, right) $S \bar{G}$-module $N$ we have $\operatorname{Hom}_{S G}(M, N) \simeq \lim _{\rightarrow} H o m_{S H}(M, N)$ (resp, $N \otimes_{S \bar{G}} M \simeq \lim N \otimes_{S \bar{H}} M$ ) where $H$ ranges over the set of finite subgroups of $G$. Then $\overrightarrow{E x t}_{S \bar{G}}^{n}(M, N) \simeq \lim _{\rightarrow} \operatorname{Ext}_{S H}^{n}(M, N)\left(\operatorname{resp}, \operatorname{Tor}_{S \bar{G}}^{n}(N, M) \simeq\right.$ $\lim _{\rightarrow} \operatorname{Tor}_{S \bar{G}}^{n}(N, M)$ ). Therefore, $s \bar{G} M$ is projective (resp, fiat).

Lemma 6. Let $R$ and $A$ be rings with local units and $F: R-\bmod \rightarrow$ $A-\bmod$ an equivalence of categories. For every $M \in R-\bmod , M$ is flat if and only if $F(M)$ is fiat.

Therefore, $w \cdot \operatorname{dim}(R)=w \cdot \operatorname{dim}(A)$

Proof: It is easy to prove that every locally projective module (see [AM] for the definition) over a ring with local units is flat. By [AM, Theorem 2.4], $F \simeq P \otimes_{R}$ - for some $A$-R-bimodule $P$ and ${ }_{A} P$ is locally projective. Thus ${ }_{A} F(M) \simeq \simeq_{A} P \otimes_{R} M$ is flat whenever $M \in R-\bmod$ is flat. The rest of the proof is obvious.

Theorem 7. Let $G$ be a locally finite group and $R=\oplus_{g \in G} R_{g}$ a $G$-graded ring. If the order of every finite subgroup of $G$ is invertible in $R_{e}$, then $\operatorname{gr.w.dim}(R)=w \cdot \operatorname{dim}(R)$.

Proof: Assume that $g r . w . \operatorname{dim}(R) \leq n$. The case $n=0$ is contained in Proposition $1(a)$ because $R_{(H)}$ has a separablity system for every finite subgroup $H$ of $G$. So we can assume $n>0$.

Consider $S=R \# G^{*}$ and $E: R-g_{r} \longrightarrow S-\bmod$ the isomorphism of categories given in (d). Since every graded flat left $R$-module is a direct limit of graded projective left $R$-modules, $E$ preserves the flatness and hence $w \cdot \operatorname{dim}(S) \leq n$.

Let $A=S \bar{G}, M \in A-\bmod$ and

$$
\mathbf{P} \equiv \ldots \stackrel{d_{3}}{\rightarrow} P_{2} \stackrel{d_{2}}{\rightarrow} P_{1} \stackrel{d_{1}}{\rightarrow} M \rightarrow 0
$$

a projective resolution of ${ }_{A} M$. Since $S A$ is projective, then $\mathrm{P}$ is also a projective resolution of ${ }_{S} M$ and hence $K=\operatorname{Ker}\left(d_{n}\right)$ is $S$-flat. By Lemma $5, K$ is also $A$-fiat. Therefore, $w \cdot \operatorname{dim}(A) \leq n$. 
Finally, let us remark that $R-\bmod$ and $A-\bmod$ are equivalent categories (see $[\mathrm{AM}$, Theorem 2.2] and [B2]). Therefore $w \cdot \operatorname{dim}(R) \leq n$.

Questions. (1) Let $R$ be a graded ring. Assume that $g r . w . \operatorname{dim}\left(R_{(H)}\right)$ $=w \cdot \operatorname{dim}\left(R_{(H)}\right)$ for every finitely generated subgroup $H$ of $G$. Does $g r . w . \operatorname{dim}(R)=w \cdot \operatorname{dim}(R)$ ?.

If $g r . w \cdot \operatorname{dim}\left(R_{(H)}\right) \leq g r . w \cdot \operatorname{dim}(R)$ for every finitely generated subgroup $H$ of $G$ (in particular if $R$ is either gr-regular or strongly graded) then the answer is yes (see the proof of Proposition 3.b). Unfortunately the example after Proposition 3 shows that $g r . w \cdot \operatorname{dim}\left(R_{(H)}\right)$ can be bigger than $g r . w . \operatorname{dim}(R)$.

(2) Is it possible to weaken the hypothesis in Theorern 7 that "the order of every finite subgroup $H$ of $G$ is invertible in $R_{e}$ " to " $R_{(H)}$ has a separability system for every finitely generated subgroup $H$ of $G$ "?

Acknowledgment. The author would like to thank Jerzy Matczuck and the referee for their helpful comments.

\section{References}

[Ab] G.D. Abrams, Morita Equivalence for Rings with Local Units, Comm. Algebra 11 (1983), 801-837.

[AM] P.N. ANH AND L. MÁRKI, Morita Equivalence for Rings without Identity, Tsukuba J. Math 11 (1987), 1-1.

[Au] M. Auslander, On Regular Group Rings, Proc. A.M.S. 8 (1957), 658-664.

[B1] M. BeatTIE, A Generalization of the Smash Product of a Graded Ring, J. Pure and Appl. Alg. 52 (1988), 219-226.

[B2] M. BEATTIE, Duality Theorems for Rings with Actions and Coactions, J: Algebra 115 (1988), 303-312.

[CE] Hi. Cartan and S. Ellenderg, "Homological Algebra," Princeton, 1956.

[CM] M. Cohen and S. Montgomery, Group-graded Rings, Smash Product and Group Actions, Trans. A.M.S. 282 (1984), 237-258.

[C] I.G. Connell, On the Group Ring, Can. J. Math. 15 (1963), 650-685.

[NV] C. NăstăsEsCU and F. VAN OYSTAEYen, "Graded Ring Theoty," North-Holland, Math. Library, 1982. 
A. DEL RÍO

[Q] D. Quinn, Group-Graded Rings and Duality, Trans. A.M.S. 292 (1985), 155-167.

[R] M.D. RAFaEl, Separable Functors Revisited, Comm. Algebra (to appear).

[V] O.E. Villamayor, On Weak Dimension of Algebras, Pac. J. Math. 9 (1959), 941-951.

\author{
Departamento de Matemáticas \\ Universidad de Murcia (Murcia) \\ SPAIN
}

Rebut el 12 de Juliol de 1989 